\title{
Tokamak Magnetic Islands in the Presence of Nonaxisymmetric Perturbations
}

\author{
A. H. Reiman \\ Princeton Plasma Physics Laboratory \\ Princeton, N. J. 08543
}

\begin{abstract}
The effects of a small, externally imposed, nonaxisymmetric magnetic field perturbation on magnetic islands are studied analytically, assuming zero $\beta$, tokamak ordering, and narrow islands. For the tearing stable case, the conditions under which the self-consistent plasma response is self-healing or amplifying are elucidated. For the tearing unstable case, the quasilinear theory of tearing modes is extended to a description of locked modes.
\end{abstract}




\section{Introduction}

A small nonaxisymmetric magnetic field can have profound effects on a tokamak equilibrium if it resonates with a low order rational surface. For a stable equilibrium, a magnetic island is produced whose width scales as the square root of the magnitude of the nonaxisymmetric field. In that case, one is interested in knowing whether self-consistent plasma currents amplify or oppose the effect. For a tearing unstable plasma, a magnetic island naturally appears even in the absence of an external perturbation, and one is then interested in knowing how a small external perturbation affects the island width. The answers to these questions are of practical interest in tolamak design, where one needs to place tolerances on allowable nonaxisymmetric field errors. On a more fundamental level, a small nonaxisymmetric field provides a probe that significantly perturbs the plasma only in a narrow region about the rational surface, allowing us to test our understanding of the physics of magnetic islands. We will also see that the presence of an iufinitesimal nonaxisymmetric perturbation provides a link between equilibrium and stability theory, suggesting an equilibrium approach to the computation of saturated tearing modes. In this paper, we study the effects of a small nonaxisymmetric perturbation analytically, assuming cylindrical geometry and narrow islands. A forthcoming paper will present three-dimensional numerical calculations. ${ }^{1}$

For the tearing stable case, a crude model of the plasma response ${ }^{2,3}$ has ronvinced many in the fusion community that tokamak plasmas are generally self healing. We will see in Sec. $V$ that this is not the case. Resonant perturbations can be strongly amplified or strongly diminished by the selfconsistent plasma response, depending on the current profile. (In fairness to the authors of references 2 and 3 , we note that their model was a reasonable first cut at a description of their experiments, and that the authors themselves made no such general claims concerning the implications of their calculations.)

The tearing unstable case is relevant to the calculation of locked modes. ${ }^{4}$ We will see that saturated island vidths can be sensitive to the presence of small perturbations. Saturated islands have previously been calculated for narrow islands in a cylinder in the absence of error fields. ${ }^{5-8}$ This is the socalled "quasilinear" theory of tearing modes. (The word "quasilinear" here refers to the approximation that only one nonaxisymmetric Fourier com- 
ponent needs to be retained in the calculation.) Our work on the tearing unstable case is an extension of this previous work to include an external perturbation.

An additional aspect of our analysis is the perspective it gives us on the calculation of saturated tearing modes. In the absence of an external perturbation, the onset of a tearing mode corresponds to a bifurcation of the axisymmetric equilibrium. Below the tearing threshold, there is a stable axisymmetric equilibrium solution. Above the threshold, there is an unstable axisymmetric equilibrium solution, and there is a nonaxisymmetric equilibrium solution corresponding to the saturated tearing mode, with the island width going to zero as the threshold is approached from above. We will see in Sec. IV that the presence of an infinitesimal nonaxisymmetric perturbation breaks the bifurcation. Below the tearing threshold, the (unique) equilibrium in the presence of the perturbation is nonaxisymmetric. This equilibrium goes smoothly into the saturated tearing mode equilibrium solution as we go through the threshold. This suggests that a natural way to calculate tearing mode effects is to track the equilibrium solution as a function of the current profile parameters in the presence of an infinitesimal nonaxisymmetric perturbation. The saturated tearing modes can be calculated directly, without following their time evolution. We will have more to say about this in Sec. VII.

In Sec. VII, we will also discuss the connection of our results with error field compensation experiments on DIII-D. ${ }^{9}$

\section{Equilibrium with : Narrow Island in the Presence of a Resonant Perturbation}

When an island width becomes greater than the width of the resistive layer, the island enters the Rutherford regime, where inertial effects may be neglected. (In practice, the width of the resistive layer is quite small, typically of the order of a milimeter.) The subsequent time development of the island occurs on the resistive time scale, with the field evolving through a sequence of equilibria as the current changes resistively. In this section we look at a snapshot at a fixed time, writing down the equation for the instantaneous equilibrium without distinguishing saturated from evolving islands. We show how the effects of an external resonant perturbation can be extracted into 
a separate term in this equation, with the dependence on the perturbation amplitude made explicit.

Our analysis assumes cylindrical geometry, with the tikamak ordering $B_{z} \gg B_{9}, a / R \ll 1$. Equilibrium quantities are functions only of $r$. Perturbed quantities are functions of $r$ and $m \theta-n \phi$. The helical symmetry allows the introduction of a helical flux function,

$$
\psi(r, m \theta-n \phi)=\psi_{0}(r)-\psi_{1}(r) \cos (m \theta-n \phi),
$$

with

$$
\mathbf{B}=\nabla \psi \times \hat{z}+\frac{n r}{m R} B_{z} \hat{\theta}+B_{z} \hat{z}
$$

The flux surfaces described by Eq. (1) have an island of width

$$
w=4\left|\psi_{1}\left(r_{s}\right) / \psi_{0}^{\prime \prime}\left(r_{s}\right)\right|^{1 / 2}
$$

at $t\left(r_{1}\right)=\pi / m$, where

$$
t=R B_{\theta} / r B_{z},
$$

and $r_{s}$ denotes the location of the rational surface.

We neglect the effects of pressure. The equilibrium equation,

$$
\mathbf{j} \times \mathbf{B}=\mathbf{0},
$$

together with the tokamak ordering gives

$$
\mathrm{j}=j(\psi) \hat{z} \text {. }
$$

The equilibrium is determined by the solution of Ampere's law:

$$
\nabla^{2} \psi=j(\psi)-\frac{2 n}{m R} B_{x}
$$

where $B_{z}$ is a constant.

In specifying the profile of $j$, it is necessary in practice to recognize the subtlety that $\psi(r)$ is not monotonic ( $\psi^{\prime}=0$ on the rational surfaces). The values of $j$ on different flux surfaces are independent constants of integration of the equilibrium equation, even if the value of $\psi$ on the two surfaces is the same. It follows that $j$ nust actually be specified as a function of $\psi$ and an additional discrete index. This complication will not be important to us in 
this paper, so we will continue to denote the functional dependence of $j$ as $j(\psi)$, with the additional dependence on a discrete index implicit.

We follow the conventional treatment of narrow islands in dividing up the domain in which we solve Eq. (4) into two regions, an exterior region where we can expand $j$ perturbatively,

$$
j(\psi)=j_{0}\left(\psi_{0}\right)-\frac{R}{r B_{1}} \frac{d j_{0} / d r}{-n / m} \psi_{1}(r) \cos (m \theta-n \phi),
$$

and a narrow jnner region near the rational surface where this approximation breaks down. It is the resonant denominator, of course, that causes the perturbation expansion to break down in the neighborhood of the rational surface where $t=n / m$. Equation (5) has been found to be a good approximation right up to the edge of the island separatrix, so our regions are as shown in Fig. 1. The regions are bounded by

$$
r_{0}=r_{s}+w-g
$$

and

$$
r_{i}=r_{s}-w-s,
$$

where

$$
s=.5\left[\psi_{1}^{\prime}\left(r_{0}\right)+\psi_{1}^{\prime}\left(r_{i}\right)\right] / \psi_{o}^{\prime \prime}
$$

The values of $r_{a}$ and $r_{i}$ can be determined in practice by iterating back and forth between equations $(6,7)$ and the equation for $s, E q .(8) .^{5}$

In the exterior region, equations (4) and (5) give the standard tearing mode equation for $\psi_{1}$,

$$
\frac{d}{d r} r \frac{d \psi_{1}}{d r}-\frac{m^{2}}{r} \psi_{1}=\frac{\jmath_{0}^{\prime} R}{B_{2}(t-n / m)} \psi_{1}
$$

We will include an external perturbation, which will enter through the boundary condition

$$
\psi_{1}(r=a)=\epsilon,
$$

where $\epsilon \cos (n \phi-m \theta)$ is the given external perturbation. There is also a regularity condition at the origin which requires that

$$
\psi_{1} \propto r^{m} \text { for } r \longrightarrow 0
$$


We take advantage of the narrowness of the interior region by integrating the equation in that region with respect to $r$ and turning it into a jump condition. Writing

$$
j(r, n \phi-m \theta)=j_{0}(r)+j_{1}(r) \cos (n \phi-m \theta)
$$

and integrating Eq. (4) across the island $\left(r=r_{i}\right.$ to $\left.r=r_{0}\right)$ gives a jump condition

$$
r_{0} \psi_{1}^{\prime}\left(r_{0}\right)-r_{i} \psi_{1}^{\prime}\left(r_{i}\right)=I_{1} \psi_{1}\left(r_{0}\right)
$$

where $w$ is the island width and

$$
I_{1}=\left[\int_{r_{i}}^{r_{o}} r j_{1}(r) d r\right] / \psi_{1}\left(r_{s}\right)+m^{2} w / r_{2} .
$$

In obtaining the $m^{2} / r$, term on the right hand side of Eq. (13), we have neglected the variation of $\psi_{1}(r)$ across the island. From Eq. (9) we see that $\psi_{1}^{\prime} / \psi_{1}$ is of order $\ln w$, so the discarded term is of order $w^{4} \ln w$. We will need the terms up to order $w^{3}$ in Eq. (12). Using the fact that $j_{1}$ is a continuous function of $\psi$; it is easily verified that $j_{1}$ is of order $w$, so that $\mathcal{I}_{1}$ is of order 1.

We rewrite the left hand side of Eq. (12), as $r_{s} \Delta_{a}^{\prime}(w) \psi_{1}\left(r_{s}\right)$, where

$$
r_{1} \Delta_{t}^{\prime}(w) \equiv\left[r_{0} \psi_{1}^{\prime}\left(r_{0}\right)-r_{i} \psi_{1}^{\prime}\left(r_{i}\right)\right] / \psi_{1}\left(r_{0}\right)
$$

is a generalization of the usual ${ }^{10} \Delta^{\prime}$ to include finite $\epsilon$ and $w$. The quantity $\Delta_{e}^{\prime}(w)$ contains the effects of $\epsilon$ through the boundary condition Eq. (10). To make this dependence explicit, and to make cortact with the usual $\Delta^{\prime}(w)$ for $\epsilon=0$, we introduce solutions $u_{i}, u_{t}$, and $u_{2}$ satisfying Eq. (9) with boundary conditions,

$$
\begin{array}{cl}
u_{1}(r=a)=0, & d u_{1} /\left.d r\right|_{r=a}=1, \\
u_{2}(r=a)=1, & d u_{2} /\left.d r\right|_{r=a}=0, \\
u_{i} \longrightarrow r^{m} \text { as } r \longrightarrow 0 .
\end{array}
$$

After some algebra we find

$$
\Delta_{c}^{\prime}(w)=\Delta^{\prime}(w)-\frac{\epsilon}{\psi_{1}\left(r_{s}\right)} \sigma^{\prime}(w),
$$


where

$\sigma^{\prime}(w)=\left\{i+\left[\frac{u_{1}^{\prime}\left(r_{0}\right)}{u_{1}\left(r_{0}\right)}+\frac{u_{i}^{\prime}\left(r_{i}\right)}{u_{i}\left(r_{i}\right)}+\frac{2}{r_{0}}-\frac{1}{4} \frac{j_{0}^{\prime}\left(r_{0}\right) R}{B_{2} t^{\prime}\left(r_{s}\right)}\right] \frac{w}{2}\right\} u_{2}\left(r_{0}\right)\left[\frac{u_{1}^{\prime}\left(r_{0}\right)}{u_{1}\left(r_{0}\right)}-\frac{u_{2}^{\prime}\left(r_{0}\right)}{u_{2}\left(r_{0}\right)}\right]$,

and the definition of $\Delta^{\prime}(w)$ is the same as that of $\Delta_{c}^{\prime}(w)(\mathrm{Eq} .(14))$ except that the boundary condition at the edge is $\psi_{1}=0$. Some of the details of the derivation are given in the appendix.

For the benefit of the reader who is unfamiliar with the literature on the quasilinear stabilization of tearing modes, we emphasize that we have three different $\Delta^{\prime}$ quantities. The first is the familiar $\Delta^{\prime}$ that determines linear tearing stability. It is standard in the work on quasilinear stabilization to introduce a nonlinear generalization of this $\Delta^{\prime}, \Delta^{\prime}(w)$, which appears in expressions for the nonlinear evolution and saturation of tearing modes. Finally, we have introduced a new quantity, $\Delta_{e}^{\prime}(w)$, which includes the effects of an imposed external magnetic field. Our definition of $\Delta^{\prime}(w)$ agrees with that of Zakharov ${ }^{8}$ but differs slightly from that of White et al, ${ }^{5}$ in that they do not include the factors of $r$. The definition we use gives a slightly more compact representation for the equations, and therefore simplifies some of the analysis. In the limit that $w \rightarrow 0$, both definitions reduce to the usual $\Delta^{\prime}$ of the linearized analysis. ${ }^{10}$ We have the relations

$$
\lim _{i \rightarrow 0} \Delta_{i}^{\prime}(w)=\Delta^{\prime}(w)
$$

and

$$
\lim _{w \rightarrow 0} \Delta^{\prime}(w)=\Delta^{\prime}
$$

We have written our equilibrium equations in terms of both $\psi_{1}\left(r_{s}\right)$ and w. We would like to use Eq. (3) to eliminate one in terms of the other. When $\varepsilon=0$, the sign of $\psi_{1}\left(r_{3}\right)$ is irrelevant, and it is possible to write everything in terms of $w$. That is what is done in the nonlinear tearing literature. When $\epsilon$ is nonzero, the sign of $\psi_{1}\left(r_{e}\right)$ relative to $\epsilon$ is important and must be retained in the equations. We therefore work in terms of $\psi_{1}\left(r_{3}\right)$. To simplify our expressions, we will define

$$
\psi_{1,} \equiv \psi_{1}\left(r_{s}\right)
$$

Equations (12), (14), and (18) give

$$
\psi_{1 s}\left[r_{s} \Delta^{\prime}\left(\left|\psi_{1 s}\right|\right)-I_{1}\left(\left|\psi_{1 s}\right|\right)\right]=\epsilon r_{s} \sigma^{\prime}\left(\left|\psi_{1 s}\right|\right)
$$


Equation (20) is our final equilibrium equation. It can be regarded as an eigenvalue equation for $\psi_{1}\left(r_{s}\right)$, and thereiore for the island width and phase, for a given current profile and a given $\epsilon$. In general, Eq. (9) is to be integrated numerically to determine the functional form of $\Delta^{\prime}\left(\left|\psi_{n_{s}}\right|\right)$ and $\sigma^{\prime}\left(\left|\psi_{1},\right|\right)$. Similarly, the value of $I_{1}$ is determined by the current profile in and near the island. Its calculation for various model $j(\psi)$ profiles is one of the main subjects of several papers on nonlinear tearing modes. ${ }^{3,8}$

\section{Time Evolution of the Island}

In the previous section we solved for the equilibrium with a magnetic island in the presence of an external perturbation. In this section we determine how that equilibrium evolves on a resistive time scale. The effects of an external perturbation appear through the boundary condition on the equilibrium. In principle, we could simply substitute our expression for $\Delta_{e}^{\prime}$ into the standard quasilinear treatments of tearing modes [references (5-8)] wherever $\Delta^{\prime}$ appears. As discussed in more detail in this section, these standard treatments of quasilinear effects are not adequate for our purposes. One widely used treatment ${ }^{6}$ substitutes $\Delta^{\prime}(w)$ for $\Delta^{\prime}$ in Rutherford's theory ${ }^{11}$ of tearing mode evolution. This approach runs into difficuity near the plasma edge, where $\Delta^{\prime}(w)$ has a nonvanishing dependence on $w$ even in the limit $j \rightarrow 0$. Zakharov has recently pointed out ${ }^{8}$ that the classic, original paper on quasilinear saturation by White et $\mathrm{al}^{5}$ neglects the quasilinear modification to the equilibrium current profile. Zakharov's paper includes this effect, but treats only the equilibrium problem. In this section we derive an evolution equation for the island which remains valid in the limit where the current density near the island gets small.

The evolution of the magnetic islands is determined by Faraday's law. Applying Eq. (1) and Ohm's law, and averaging over the flux surfaces to eliminate $\checkmark \cdot \nabla \psi$, we get ${ }^{5.7}$

$$
\left\langle\frac{\partial \psi}{\partial t}\right\rangle=-\eta j(\psi)+E,
$$

where $E$ is a constant determined by the electric field at the boundary, and 
where the flux surface average is defined by

$$
\langle f\rangle \equiv \int f \mathcal{J} d \theta d \phi \mid \int \mathcal{J} d \theta d \phi
$$

with the Jacobian $\mathcal{J}=(\nabla \psi \cdot \nabla \theta \times \nabla \phi)^{-1}$. We have assumed that the resistivity, $\eta$, is a function of the flux surface. This is appropriate because the time scale for temperature equilibration on a flux surface is determined by the electron thermal velocity, and is fast compared to the time scale on which the island evolves.

Equation (21) gives us a relation between $j_{1}$ and $\partial \psi / \partial t$ in the interior region. Substituting into the equilibrium equation, Eq. (20), via Eq. (13), we get an evolution equation that can be solved order by order for the time dependence of the island,

$$
\begin{aligned}
& \frac{1}{\pi} \int_{r_{i}}^{r_{0}} r\left[\int_{0}^{i \pi} \cos (\zeta) \frac{1}{\eta}\left\langle\frac{\partial \psi}{\partial t}\right\rangle d \zeta\right] d r= \\
& -\psi_{1,}, r_{0} \Delta_{\varepsilon}^{\prime}\left(\left|\psi_{1 s}\right|\right)-\frac{E}{\pi} \int_{r_{i}}^{r_{0}} r\left[\int_{0}^{2 \pi} \cos (\zeta) \frac{1}{\eta} d \zeta\right] d r-m^{2} \frac{w}{r_{s}} \psi_{1},
\end{aligned}
$$

where

$$
\zeta \equiv n \phi-m \theta \text {. }
$$

(The quasilinear modification of $\psi_{0}$ must be incorporated in evaluating the average over a flux surface in all orders above the lowest. ${ }^{8}$ )

To lowest order

$$
\partial \psi / \partial t \approx-\dot{\psi_{1}}\left(r_{3}\right) \cos (m \theta-n \phi),
$$

and Eq. (22) gives a generalization of Rutherford's result: ${ }^{11,7}$

$$
\begin{aligned}
\frac{\partial \psi_{1\lrcorner}}{\partial t} & =\pi \eta_{x} \Delta_{t}^{\prime}(w=0) \psi_{1 s} / w \\
& =\pi \eta_{x}\left[\Delta^{\prime} \psi_{1},-\epsilon \sigma^{\prime}\right] / w
\end{aligned},
$$

where $\eta_{x}$ is the value of $\eta$ at the $X$-point. This is consistent with the equation obtajned by Boozer ${ }^{12}$ for the evolution of a magnetic island in the presence of a given fixed resonant field perturbation at a rational surface. A similar 
expression was also obtained by Hahm and Kulsrud for a slab geornetry with a flat current profile $\left(j^{\prime}=0\right)$ and a perturbed boundary. ${ }^{13}$

Equation (23) breaks down when $\epsilon / \psi_{1}$, is sufficiently large. Inertial effects become important, even with the island width greater than the width of the resistive layer. In this paper we will be mainly interested in solving for the steady-state solutions and their stability, so this case will not be of concern.

Equation (23) is adequate to determine the steady-state response to an external perturbation for the tearing stable case. For that case

$$
\psi_{1}\left(r_{9}\right) \approx \epsilon \sigma^{\prime}(w=0) / \Delta^{\prime}(w=0) .
$$

As we approach the threshold for tearing instability, $\Delta^{\prime} \rightarrow 0$, the next order term becomes important. It is this term that causes saturation when the tearing mode is unstable.

From Eq. (21) it follows that we can write

$$
\int_{r_{i}}^{r_{0}} r j_{1} d r=a w \psi_{1}+b w \psi_{1},
$$

where $a$ and $b$ are coefficients to be determined. In obtaining Eq. (23), we have evaluated $a$ to lowest order:

$$
a \approx \frac{r_{2}}{\pi \eta_{x}}\left(1+a_{1} w\right)
$$

Substituting equations (24) and (25) into equations (13) and (20) gives

$$
\left(1+a_{1} w\right) \dot{\psi}_{1}=\eta_{x} \pi \psi_{1}\left[\Delta^{\prime}(w)-b w / \tau_{s}-m^{2} w / r_{:}^{2}\right]-\eta_{x} \pi \epsilon \sigma^{\prime}(w) .
$$

The coefficien ts $a_{1}$ and $j$ are independent of $\epsilon$. Numerical evaluation for $c=0$ has shown that $a_{1}$ and $b$ can generally be neglected relative to the $w$ dependence of $\Delta^{\prime}(w) .6$ However, this does not give a reasonable answer in the limit where the current density goes to zoro in the vicinity of the island. When the rational surface lies outside the plasma, for example, the growth of the island cannot lead to any local redistribution of current, so that the finite $w$ corrections to Eq. (23) should vanish. An evaluation of $\Delta^{\prime}(w)$ for this case gives

$$
\Delta^{\prime}(w)=\Delta^{\prime}+2 w m^{2} / r^{2}
$$

The resolution of this apparent paradox comes from the observation that $b=m^{2} / r_{1}$ in this case. ${ }^{5,8}$ In the limit that $j_{0} \rightarrow 0$, the finite $w$ dependence of 
$\Delta^{\prime}(w)$ is exactly canceled by two other terms: the $b$ term, and the $m^{2} w / r_{\text {s }}^{2}$ term in the expression for $I_{1}$. For the numerical evaluations of Ref. 6 , the rational surface is in the interior of the current distribution, and these terms can be neglected. To obtain an expression which is also valid near the plasma edge, we retain these terms. Our final equation describing the evolution of the island is

$$
\frac{\partial \psi_{1 s}}{\partial t}=\frac{\pi \eta_{x}}{w}\left\{\psi_{1 \cdot}\left[\Delta^{\prime}(w)-2 \frac{m^{2}}{r_{1}^{2}} w\right]-\epsilon \sigma^{\prime}(w)\right\} .
$$

Steady-state islands are described by the equation

$$
\left[\Delta^{\prime}(w)-2 w m^{2} / r_{9}^{2}\right] \psi_{1,}=\epsilon \sigma^{\prime}(w) .
$$

\section{Steady-State Solutions and Their Stability}

In this section we will discuss the steady-state solutions of Eq. (27), and their stability. We will begin by describing the general qualitative features of the solution, leaving detailed descriptions and proofs for later in the section. We proceed in this way to give the reader an overall picture of what the solution looks like before getting into the details of the algebra. Also, we want to emphasize the generic nature of the main properties of the solution. Many of the significant properties of the solution follow from general arguments of bifurcation theory, ${ }^{14}$ and do rot depend on the details of the equations. To make this clear, and to develop some additional intuitive understanding, we will also discuss an analogous model of a ball in a potential well.

For reference, let us first consider the case $\epsilon=0$ (no exterral perturbation). Figure 2 shows a schematic plot of $\psi_{1}$, as a function of a profile parameter, which we will call $\gamma$. We assume that $\Delta^{\prime}$ increases monotonirally with $\gamma$, and that $\gamma=0$ corresponds to $\Delta^{\prime}=0$. Of course, the other coefficients in Eq. (28) will also be functions of $\gamma$. For $\gamma<0$ there is a unique solution, corresponding to a cylindrically symmetric, stable equilibrium. For $\gamma>0$, the cylindrically symmetric equilibrium is unstable. There are also solutions corresponding to saturated tearing nodest. These are stable, nonsymmetric equilibria with islands. As $\gamma \rightarrow 0$ from the right, the island width goes to zero. The point $\gamma=0$ corresponds to a bifurcation point of the solution.The equations are independent of the sign of $\psi_{1 s}$, so Fig. 2 has up-down 
symmetry. There is an additional degree of fretciom not shown in this figure: the phase of $\psi_{1}$ is completely arbitrcry.

Analogous situations for a ball in a putential well are shown in figures 3 and 4. To model the arbitrariness in the phase of $\psi_{1}$, we thiuk of each of these figures as a two-dimensional slic, through a three-dimensional picture, with the potential well symmetric with respect to rotation about its center. Figure 3 corresponds to $\gamma<$ ?. There is a unioge, stable equilibrium. Increasing $\gamma$ corresponds to raising the potential at the center. Figure 4 corresponds to $y>0$. The "synmetis"ce equilibrium, in which the ball sits at the center of the poiential wsil, is unstable. There are now also "nonsymmetric" equilibrium solutions, at: $d$ they are stable.

When $\therefore$, the soltition of Eq. (28) is as shown in Fig. 5. There is still a unique, statle solntion for $\gamma<0$, but the equilibrium is now a nonsynmetric cne witt an island. For larye $\gamma$ th.cre are again three solutions. The externai perturtation breaks the bifurcation at $\gamma=0$, so that the solution now consists of two independent branches. The branch that we are interested in is the one that extends from $\gamma<0$.

To extend the analogy of the ball in a potential well to nonzero $\epsilon$, we impose an additional uniform force in the negative $x$ direction. The force breaks the rotational symmotry of the potential well. Figures 6 and 7 show the corresponding situations, with the arrow indicating the direction of the additional uniform force. "igure 6 corresponds to $\gamma<0$. The time-independent solution is unique, and it is a stable equilibrium which is no longer located at $x=0$. Figure 7 cursesponds to $\gamma>0$. The balls in the figure are locater at the three tune-indeperdent sclutions.

The middle bail in Fig. 7 is in an unstable equilibrium. Any small motion aloug the $x$ axis is amplified. The two balls on the end are stable with respect to perturbations aiong $x$. However, the ball at the far right is unstable with respect to motions out of the piane. The ball at the far left is in a stable squilibrium. The stability properties are the same as those for a magnetic island, and are summarized in Fig. 5.

Now let's see how these properties come ont of equations (28) and (27). For $w$ small, we write

$$
\Delta^{\prime}(w) \approx \Delta^{\prime}+2 w m^{2} / r_{s}^{2}-a w,
$$


and

$$
\sigma^{\prime}(w) \approx \sigma_{\mathrm{G}}^{\prime}+\sigma_{1}^{\prime} w .
$$

We will assume that $\sigma_{0}^{\prime}$ is nonzero, so that the $\sigma_{1}^{\prime} w$ correction can be neglected for to small. The $\alpha w$ term cannot be neglected because $\Delta^{\prime}=0$ for $\gamma=$ 0. (When $\Delta^{\prime}$ is small, $\epsilon \sigma_{0}^{\prime}$ balances $\alpha w \psi_{10}$, so that $\epsilon \sigma_{1}^{\prime} w$ is of order $w^{4}$.) Rewriting the equation in terms of $w$ and carrying the sign of $\psi_{1} s / \psi_{0}^{\prime \prime}\left(r_{s}\right)$ over to the right hand side of the equation, we get

$$
\mathcal{C}^{\prime} u^{2}-\alpha w^{3}= \pm \bar{\epsilon}
$$

where

$$
\bar{\epsilon}=16 \sigma_{0}^{\prime} \epsilon / \psi_{0}^{n i}\left(r_{s}\right) .
$$

The sign on the right hand side of Eq. (31) zorresponds to that of $\psi_{1 s} / \psi_{0}^{\prime \prime \prime} r_{s}$ ), which may be either positive or negative. We will take $\bar{\varepsilon}>0$, without loss of generality. (We are free to choose the sign of $\epsilon$.)

We assume throughout this section that the finite $w$ correction to $\Delta^{\prime}$ is stabilizing $(\alpha>0)$. If that is not the case, there are no saturated tearing modes within our theory. Unstable tearing modes grow until higher order nonlinearities become important. For finite $\epsilon$, the island width becomes large as $\Delta^{\prime}$ approaches zero from below. We will discuss the case $\alpha=0$ in Sec. $V$.

Figure 8 shows a plot of $\Delta^{\prime} w^{2}-\alpha w^{3}$ vs. $w$ for $\Delta^{\prime}<0, \alpha>0$. The shaded region corresponds to $w<0$, which is of no interest. It is clear that there is a unique equilibrium solution for any value of $\epsilon$. For $\Delta^{\prime}$ large and negative, the island width scales like $\epsilon^{1 / 2}, w \approx\left(-\bar{\varepsilon} / \Delta^{\prime}\right)^{1 / 2}$. As $\Delta^{\prime} \rightarrow 0$ we get $w \rightarrow(\bar{\epsilon} / \alpha)^{1 / 3}$

Figure 9 shows a p.ot of $L^{\prime} w^{2}-\alpha w^{3}$ vs. $w$ for $\Delta^{\prime}>0, \alpha>\eta, w \geq 0$. There are three roots when $\left(\Delta^{\prime}\right)^{3}>27 \alpha^{2} \bar{\epsilon} / 4$. For small $\epsilon$ we ha $>$ roots at $w \approx \Delta^{\prime} / \alpha$ and $w \approx\left(\bar{\epsilon} / \Delta^{\prime}\right)^{1 / 2}$. Two of the roots coalesce at $w=: \Delta^{\prime} / 3 \alpha$ for $\left(\Delta^{\prime}\right)^{3}=27 \alpha^{2} \vec{c} / 4$. There is a single soot at smaller values of $\Delta^{\prime}$.

To determine the stability of the steady-state solutions, let us rewrite Eq. (27) using equations (29) and (30):

$$
2 w^{2} d w / d t=\pi \eta_{x}\left(\Delta^{\prime} w^{2}-\alpha w^{3} \div \bar{\epsilon}\right),
$$

where again the $\mp$ sign corresponds to that of $\psi_{1} / \psi_{0}^{\prime \prime}\left(r_{\mathrm{s}}\right)$. The sign of the right hand side of Eq. (33) can be read off the graphs of figures 8 and 9 . 
Consider first $\Delta^{\prime}<0$ (Fig. 8). In steady-state, the island width satisfies $\Delta^{\prime} w^{2}-\alpha w^{3}+\bar{\varepsilon}=0$. Examination of Fig. 8 shows that for larger island widths the right hand side of Eq. (33) is negative, while, for smaller island widths the right hand side is positive. This steady-state solution is stable to a perturbation of $w$. For $\Delta^{\prime}>0$ (Fig. 9) the small $w$ steady-state solution is unstable, while the other steady-state solutions are stable to perturbation of $w$.

In addition to perturbations of $w$, we also need to consider perturbations which rotate the island slightly relative to the fixed external nonaxisymmetric boundary condition. In that case the external field is slightly out of phase with the internal plasma currents, and the plasma is no longer in equilibrium. A calculation of the resulting force ${ }^{15,16}$ shows that it is proportional to $\sin (m \theta)$, where $\theta$ is the angle between the rotated magnetic island and the island produced by the external feld. The solution in which the external field increases the island width is stable, while that in which the external field causes the island width to decrease is unstable.

\section{Self-Healing and Amplification: A Simplified Model}

In the previous section we discussed some general properties of the steady. state magnetic island solutions in the presence of an external perturbation. In this section, we take a closer look at the solutions for $\Delta^{\prime}<0$, focusing on a question that is of particular interest: under what conditions is the plasma response self-healing or amplifying? For this purpose we make a further simplification in our model, assuming that the island lies in the vacuum region outside the plasma. No other assumptions are made concerning the current profile.

We determine the island width from equations $(31,32)$. With the island in the vacuum region, $u_{1}$ and $u_{2}$ can be explicitly determined for $r \geq r_{\text {a }}$ by solving Eq. (9) with $j_{0}^{\prime} \rightarrow 0$, giving

$$
\sigma_{0}^{\prime}=\frac{2 m}{r_{s}} \frac{1}{\left(r_{s} / a\right)^{m}-\left(r_{s} / a\right)^{-m}} .
$$

The $\alpha$ coefficient vanishes, yielding an explicit expression for the island width,

$$
w^{2}=-16 \frac{\epsilon}{\psi_{0}^{\prime \prime}}\left(\frac{r_{s}}{a}\right)^{m} \frac{\Delta_{v}^{\prime}}{\Delta^{\prime}},
$$


where

$$
\Delta_{v}^{\prime}=\frac{2 m}{r_{*}} \frac{1}{\left(r_{d} / a\right)^{2 m}-1}
$$

is $\Delta^{\prime}$ for $j_{0}^{\prime} \longrightarrow 0$ in Eq. (9).

For $\Delta^{\prime}=\Delta_{v}^{\prime}$, we get the usual error field in vacuum. We can get amplification or self-healing, depending on whether $\left|\Delta^{\prime}\right|$ is greater than or less than $\left|\Delta_{v}^{\prime}\right|$. This result is intuitively reasonable. When $\Delta^{\prime}$ is sufficient' $y$ large and negative, the plasma is self-healing. When $\Delta^{\prime}<0$ approaches zero, we get amplification. There is a critical value of $\Delta^{\prime}$ at which the island width is just that due to the vacuum error field. Figure 10 is a plot of $\Delta_{v}^{\prime} a$ vs $r_{3} / a$ for an $m=2$ island.

Let us examine the calculations reported in references 2 and 3 in the light of these results. Those calculations employed a model that represents the plasma current by a single current filament. The plasma response is modeled by allowing the current filament to follow the magnetic axis. In calculations with this model, the islands at the $q=2$ and $q=3$ surfaces were found to be much smaller than those computed with an axisymmetric current filament.

We note, first, that the magnetic axis is unaffected by a pure $m=2$ or $m=3$ perturbation, or by any perturbation consisting only of poloidal mode numbers greater than one. Although we have found that a tokaniak can either amplify or diminish the amplitude of such a perturbation, the filament model does not incorporate this self-consistent effect.

The experiment on the ST tokamak included a substantial $m=1$ perturbation, giving a large deformation of the magnetic axis. A filamentary current has $q<1$ at the edge of the current (in fact $q \rightarrow 0$ as the width of the filament goes to zero). It follows that $m=1$ modes are kink unstable, and self-consistent effects should reinforce any $m=1$ perturbations. The calculations reported in Ref. 2 consider only $m=2$ and $m=3$ jslands. These island widths are substantially decreased by the helical deformation of the filament. This result may indicate that the plasma response decreases the coupling between $m=1$ and higher $m$ perturbations. Even this inference is unclear, given the expected sensitivity of this result to the $q$ profile, and given the fact that $q \approx 0$ for the filament case.

The model of references 2 and 3 was a reasonable first cut at a description of their experiment. A more sophisticated analysis indicates that its 
conclusions have to be interpreted with care. $A$ conciusion that plasmas are generally self-healing is unwarranted. The authors themselves made no such general claims concerning the implications of their calculation.

\section{A Slab Model}

In this section we explicitly calculate the effects of a nonsymmetric perturbation for an analytically soluble slab model. To go from cylindrical geometry to slab geometry, we make the substitutions $r \rightarrow x, m \theta-n \phi \rightarrow k y$. The flux function is of the form

$$
\psi(x, y)=\psi_{0}(x)-\psi_{1}(x) \cos (k y),
$$

with $\psi_{0}$ and $\psi_{1}$ even in $x$. The magnetic field is

$$
\mathbf{B}=\nabla \psi \times \dot{z}+B_{2} \dot{z} .
$$

In the tearing mode equation for the exterior region, Eq. (9), we substitute the expression for $\nabla^{2}$ in a slab. To maintain the symmetry of $\psi_{1}$, we impose our perturbed boundary condition in a symmetric fashion:

$$
\psi_{1}(x= \pm a)=\epsilon / 2
$$

In deriving, our relation between $\Delta_{\tau}^{\prime}$ and $\Delta^{\prime}$, eqs. (18) and (19), we used boundary conditions appropriate to cy]indrical geometry [Eq. (17)]. In slab geometry we dispense with $u_{i}$. We use Eqs. (15) and (16), and we follow the same steps as in the appendix to obtain Eq. (18) with

$$
\sigma^{\prime}=u_{2}(0)\left[\frac{u_{1}^{\prime}(0)}{u_{1}(0)}-\frac{u_{2}^{\prime}(0)}{u_{2}(0)}\right] \text {. }
$$

To first order, $\sigma^{\prime}$ is independent of $w$.

We will use the current profile of Furth et al, ${ }^{10}$

$$
j_{0}=-(b / l) \operatorname{sech}^{2}(x / l) \text {. }
$$

The solution to the tearing mode equation in the exterior region is

$$
\psi_{1}(x, y)=C e^{-k x}\left[1+\frac{\tanh (x / l)}{k l}\right]+D e^{k x}\left[1-\frac{\tanh (x / l)}{k l}\right],
$$


where $C$ and $D$ are constants to be determined from the boundary conditions.

We simplify the calculation of $\Delta^{\prime}(w)$ and $\sigma^{\prime}$ by assuming $k a \gg 1$. To first order is: $w$, we get

$$
\Delta^{\prime}(w) \approx-\frac{2}{k l^{2}}\left(k^{2} l^{2}-1\right)+\frac{2 w}{l^{2}}\left(k^{2} l^{2}-2\right) .
$$

in calculating $u_{1}^{\prime}(0) / u_{1}(0)-u_{2}^{\prime}(0) / u_{2}(0)$, the lowest order term in $\exp (-k a)$ cancels. The next order term gives

$$
\sigma^{\prime} \approx-2 \frac{k_{1}+1}{l} e^{-k_{a}} .
$$

Figure 11 shows a plot of the resulting island width as a function of $1 /(k l)$. For each of the curves, we have fixed the value of the vacuum island width, that is, the island width in the absence of the self-consistent plasma respo:se,

$$
w_{v} \equiv 4\left(e^{-k_{\sigma}} \epsilon / \psi_{0}^{\prime \prime}\right)^{1 / 2} .
$$

The slab equilibrium is tearing stable for $k l>1$, and tearing unstable for $k !<i$ This is reflected in the $w_{v}=0$ curve, which has $w=0$ when the equilibrium is stable, and has the finite saturated island width for the unstable tearing modie. In the large $k l$ limit, $w \rightarrow w_{v}$ for each of the curves. For the finite $w_{v}$ cases, the perturbation is amplified for finite values of $k l$, with the amplification becoming substantial in the neighborhood of the tearing threshold. In the small $k l$ limit, $w$ asymptotically approaches the $w_{v}=0$ saturated island width.

At the instability threshold, $w \approx .097 \approx 3.2 w_{v}$ for $w_{v}=.03$, corresponding to an amplification of over an order of magnitude for $\psi(x=0)$. This amplification will depend on the current profile. When $j_{\mathrm{a}}$ is smail at the rational surface, $\alpha$ is also small, and the amplification gets large. In the limit that $j_{0}\left(r_{0}\right) \rightarrow 0$, the instability goes over to an external kink, and the nonlinear saturation due to the local modification of the curre?t profile vanishes.

For our slab model, the external perturbation is always am:lified. We saw in the previous section that this is not always the case, that in general we can get either amplification or self-healing. 


\section{Discussion}

In the absence of an external perturbation, the equilibrium solutions in a cylinder bifurcate at the tearing threshold (Fig. 2). Below the threshold there is a stable cylindrically symmetric solution. Above the threshold, the cylindrically symmetric equilibrium is unstable to tearing modes. The saturated tearing mode corresponds to an equilibrium sulution with islands, which branches off from the cylindrically symmetric equilibrium at the tearing threshold.

An external perturbation breaks the bifurcation at the tearing threshold (Fig. 5). There is again a unique solution at $\Delta^{\prime}<0$, now corresponding to a nonsymmetric solution with an island. This stable equilibrium solution goes continuously into the stable equilibrium solution for $\Delta^{\prime}>0$.

The breaking of the bifurcation suggests an alternative approach to the calculation of saturated tearing modes (Fig. 12). The conventional approach, indicated in Fig. 12a, starts with an unstable equilibrium solution, and follows the growth and saturation of the island. An alternative approach, indicated in Fig. 12b, is to add a smail perturbation and track the equilibrium solution from the $\Delta^{\prime}<0$ region into the $\Delta^{\prime}>0$ region. This approach has the virtue of allowing the direct calculation of saturated island widths for a range of plasma parameters.

For $\Delta^{\prime}<0$, we have employed a simplified model to study the conditions under which the plasma response to an external perturbation is self-healing or amplifying. This model assumes that the island lies in the vacuum region outside the plasma, but makes no further assumptions concerning the current profile. We find that for sufficiently large and negative $\Delta^{\prime}$ the plasma response is self-healing. In some region below $\Delta^{\prime}=0$ the plasma amplifies the perturbation. There is a critical value of $\Delta^{\prime}$ (given by Eq. (36)) that marks the boundary between the two regions. For this value of $\Delta^{\prime}$ the island width is just what it would be in the absence of the self-consistent plesma response.

As $\Delta^{\prime}$ approaches zero from below, the amplification can become large. This is to be contrasted with the case where there is no external perturbation, when the island width is zero for $\Delta^{\prime} \leq 0$. The presence of an error field in a tokamak broadens the range of parameters over which deleterious effects due to tearing are seen. 
This conclusion is consistent with the results of error field compensation experiments on DIII-D. ${ }^{9}$ A coil that is capable of partially cancelıng the error fields has been placed on the tokamak. The threshold for the low density locked mode has been found to vary systematically with the amplitude of the current in the error compensation coil. The lowest densities are obtained with a finite current in the coil, corresponding to a partial cancieiaticn of the field artors. Increasing or decreasing the coil current from this optimum value increases the density threshold.

\section{ACKNOWLEDGMENT}

I am indebted to R. Goldston, D. Monticello, N. Pomphrey, W. Stodiek, and L. Zakharov for useful discussions. This work was supported by the United States Department of Energy under Contract DE-AC02-76-CHO3073. 


\section{Appendix. Relation Between $\Delta_{\epsilon}^{\prime}(w)$ and $\Delta^{\prime}(w)$}

The quantity $\Delta_{c}^{\prime}(w)$ introduced in Sec. Il is a generalization of the usual $\Delta^{10}$ to include finite $\epsilon$ and $w$. It contains the effects of $\epsilon$ through the boundary concition Eq. (10). We can make the dependence explicit by expressing $\Delta_{c}^{\prime}(w)$ in terms of the usual nonlinear $\Delta^{\prime}(w)$ for $c=0.5 .7 .8$ For this purpose we introduce solutions $u_{i}, u_{1}$, and $u_{2}$ satisfying Eq. (9) with boundary conditions given by equations (15-17). We can then write $\psi_{1}$ in the form

$$
\psi_{1}= \begin{cases}a_{1} u_{1}+\epsilon u_{2} & \text { for } r>r_{0}, \\ a_{i} u_{i} & \text { for } r<r_{i} .\end{cases}
$$

We have

$$
r_{0} \psi_{1}\left(r_{s}\right) \Delta_{c}^{\prime}(w)=r_{0} \epsilon u_{2}^{\prime}\left(r_{0}\right)+r_{0} a_{1} u_{1}^{\prime}\left(r_{0}\right)-r_{i} a_{i} u_{i}^{\prime}\left(r_{i}\right) .
$$

We can similarly write

$$
r_{s} \psi_{1}\left(r_{s}\right) \Delta^{\prime}(w)=r_{00} a_{10} u_{1}^{\prime}\left(r_{\infty 0}\right)-r_{i 0} a_{i 0} u_{i}^{\prime}\left(r_{i 0}\right) .
$$

In these expressions, $w$ is regarded as an independent variable, so $\psi_{1}\left(r_{1}\right)$ has a fixed value determinec by Eq. (3). We have

$$
\begin{aligned}
\psi_{1}\left(r_{s}\right) & \approx a_{i} u_{i}\left(r_{i}\right)+\frac{w}{2} \psi_{a}^{\prime \prime} s \\
& \approx a_{i 0} u_{i}\left(r_{i 0}\right)+\frac{w}{2} \psi_{0}^{\prime \prime} s_{0},
\end{aligned}
$$

where

$$
\psi_{0}^{\prime \prime} s=\frac{1}{2}\left[a_{i} u_{i}^{\prime}\left(r_{i}\right)+a_{1} u_{1}^{\prime}\left(r_{0}\right)+\epsilon u_{2}^{\prime}\left(r_{0}\right)\right],
$$

and

$$
\psi_{0}^{\prime \prime} s_{0}=\frac{1}{2}\left[a_{i 0} u_{i}^{\prime}\left(r_{i 0}\right)+a_{10} u_{1}^{\prime}\left(r_{\infty}\right)\right] .
$$

We can also express $\psi_{1}\left(r_{a}\right)$ in terms of $u_{1}$ and $u_{2}$,

$$
\begin{aligned}
\psi_{1}\left(r_{s}\right) & \approx a_{1} u_{1}\left(r_{0}\right)+\epsilon u_{2}\left(r_{0}\right)-\frac{w}{2} \psi_{0}^{\prime \prime} s \\
& \approx a_{10} u_{1}\left(r_{o 0}\right)-\frac{w}{2} \psi_{0}^{\prime \prime} s_{0} .
\end{aligned}
$$


Taylor expanding, and using Eq. (9), we get the relation

$$
\left(r_{0}+\delta r\right) u^{\prime}\left(r_{0}+\delta r\right) \approx r_{0} u^{\prime}\left(r_{0}\right)+\delta r \frac{j_{0}^{\prime} R}{B_{z} t^{\prime} w} u
$$

This gives

$$
\begin{aligned}
r_{0} \psi_{1}\left(r_{s}\right) \Delta_{c}^{\prime}(w) \approx & r_{0} \psi_{1}\left(r_{0}\right) \Delta^{\prime}(w)+r_{0} \epsilon u_{2}^{\prime}\left(r_{0}\right) \\
& +r_{0} \delta a_{1} u_{1}^{\prime}\left(r_{0}\right)-r_{i} \delta a_{i} u_{i}^{\prime}\left(r_{i}\right)-\psi_{1}\left(r_{s}\right) \frac{j_{0}^{\prime} R}{B_{z} t^{\prime} w}\left(\delta r_{i}+\delta r_{0}\right) .
\end{aligned}
$$

with the definitions

$$
\delta a \equiv a-a_{0}
$$

for each of the coefficients, and

$$
\delta r \equiv r-r_{0}
$$

Equations (6) and (7) give

$$
\delta r_{0}=\delta r_{i}=\delta s
$$

Expanding Eq. (A3), and retaining terms up to order $w^{3}$, we find

$$
\varepsilon a_{i} u_{i}\left(r_{\bullet} !=-w \psi_{0}^{\prime \prime} \delta s / 2\right.
$$

Equation (A6) gives

$$
\delta a_{1} u_{1}\left(r_{0}\right)=-\epsilon u_{2}\left(r_{0}\right)+w \psi_{0}^{\prime \prime} \delta s / 2 .
$$

Substituting equations ( $A 10, A 1:)$ into equations $(A 4, A 5)$, we find

$$
\psi_{0}^{\prime \prime} \delta s=\epsilon\left[u_{2}^{\prime}\left(r_{0}\right)-u_{2}\left(r_{0}\right) u_{1}^{\prime}\left(r_{0}\right) / u_{1}\left(r_{0}\right)\right] .
$$

Finally, we substitute equations (A9-A12) into Eq. (A8) to get equations (18) and (19). 


\section{References}

${ }^{1}$ A. Reiman and D. Monticello, Sensitivity of Tokamak Equilibria to the Presence of Nonaxisymmetric Error Fields - Numerical Computations, to be published, 1990.

${ }^{2}$ W. Stodiek, K. Bol, H. Eubank, S. V. Goeler, and D. J. Grove, in Plasma Physics and Controlled Nuclear Fusion Research, Proceedings of the Fourth International Conference, Madison, Vol. I, pages 465-478, 1971.

${ }^{3}$ R. Goldston, S. Davis, H. Eubank, R. Hawryluk, D. Johnson, D. McCune, D. Mueller, J. Schivel!, G. Schmidt, F. Stauffer, H. Towner, the PLT. and N. B. Groups, in Heating in Toroidal Plasmas, pages 711-721, Commission of the European Communities, September 1980, Proceedings of the 2nd Joint Grenoble-Varenna International Symposium - Villa Olmo Como - Italy.

'J. A. Snipes, D. J. Campbell, P. S. Haynes, T. C. Hender, M. Hugon, P. J. Lomas, N. J. L. Cardozo, M. F. F. Nave, and F. C. Schueller, Nucl. Fusion 28(6), 1085-1097 (1988).

${ }^{5}$ R. B. White, D. A. Monticello, M. N. Rosenbluth, and B. V. Waddell, Phys. Fluid 20, 800 (1977).

${ }^{6}$ B. Carreras, B. Waddel, and H. R. Hicks, Nucl. Fusion 19, $1423(1979)$.

${ }^{7}$ R. B. White, Rer. Mod. Phys. 58, 183 (1986).

${ }^{8}$ L. Zakharov, Quasilinear Equilibria Related to Tearing Modes in Tokamaks, Technical Report FRCR 319, Fusion Research Center, University of Texas, Austin, Texas, October 1988.

${ }^{9}$ T. Scoville, R. LaHaye, A. Kellman, T. Osborne, R. Stambaugh, E. Strait, and T. Taylor, Prevention of the $n=1$ locked mode in DiII-D Plasmas, Bull. APS 34(9), 2118 (October 1989).

${ }^{10}$ H. P. Furth, J. Killeen, and M. N. Rosenbluth, Phys. Fluids 6, 459 (1963).

${ }^{11}$ P. H. Rutherford, Phys. Fluids 16, 1903 (1973). 
${ }^{12}$ A. H. Boozer, Phys. Fluids 27(8), 2055-2062 (August 1984).

${ }^{13}$ T. S. Hahm and R. M. Kulsrud, Phys. Fluids 28(8), 2412-2418 (A ugust 1985).

${ }^{14}$ G. Ioos and D. D. Joseph, Elementary Stability and Bifurcation Theory, Springer-Verlag, New York, gecond edition, 1990.

${ }^{15}$ T. C. Hender, C. G. Gimblett, and D. C. Robinson, in isth European Conference on Controlled Fusion and Plesma Heating, edited by S. Pesic and J. Jacquinot, pages 437-440, Dubrovnik, May 1988, European Physical Society, Volume 12B, part I.

${ }^{16} \mathrm{H}$. Zohm, A. Kallenbach, H. Bruhys, G. Fussmasin, and O. Klueber, Europhys. Lett. 11(8), 745-750 (1990). 


\section{Figures}

FIC. 1. Division of the domain into regions for the solution of Ampere's law. The interior region is stippled. It is further divided into subregions $I$ and II, separated by the separatrix.

FIG. 2. Schernatic plot of $\psi_{1}\left(r_{0}\right)$ as a function of a profile parameter $\gamma$ for $\epsilon=0$. We assume that $\Delta^{\prime}$ increases monotonically with $\gamma$.

FIG. 3. Analogous model of a ball in a potential well. The figure shown corresponds to $\gamma<0$

FīG. 4. Model of ball in a potential well corresponding to $\gamma>0$.

FIG. 5. Solution for $\psi_{1}\left(r_{1}\right)$ with finite $\epsilon$. The dashed line indicates the solution in the $\epsilon=0$ limit.

FIG. 6. Ball in a potential well with an additional uniform force imposed. This case correspends to $\gamma<0$. The arrow indicates the direction of the additiona! force. The ball is in a stable equilibrium.

FIG. 7 . Balls in a potential well with additional uniform force, $\gamma>0$. Locations of the balls indicate the three time-independent solutions.

FIG. 8. Plot of the steady-state equation for $\Delta^{\prime}<0$.

FIG. 9. Plot of the stead $y$-state equation for $\Delta^{\prime}>0$.

FIG. 10. Plot of $\Delta_{v}^{\prime} a$ vs $r_{3} / a$ for an $m=2$ island lying outside the plasma.

FIG. 11. Island width for a slab model with $j_{0} \propto \mathrm{sech}^{2}$. For each curvit, the vacuum island width $w_{v}$ is held fixed.

FIG. 12. Schematic view of saturated island calculation by a) conventional time-dependent methods, and b) tracking the equilibrium solution. 
$91 \mathrm{~T} 1001$

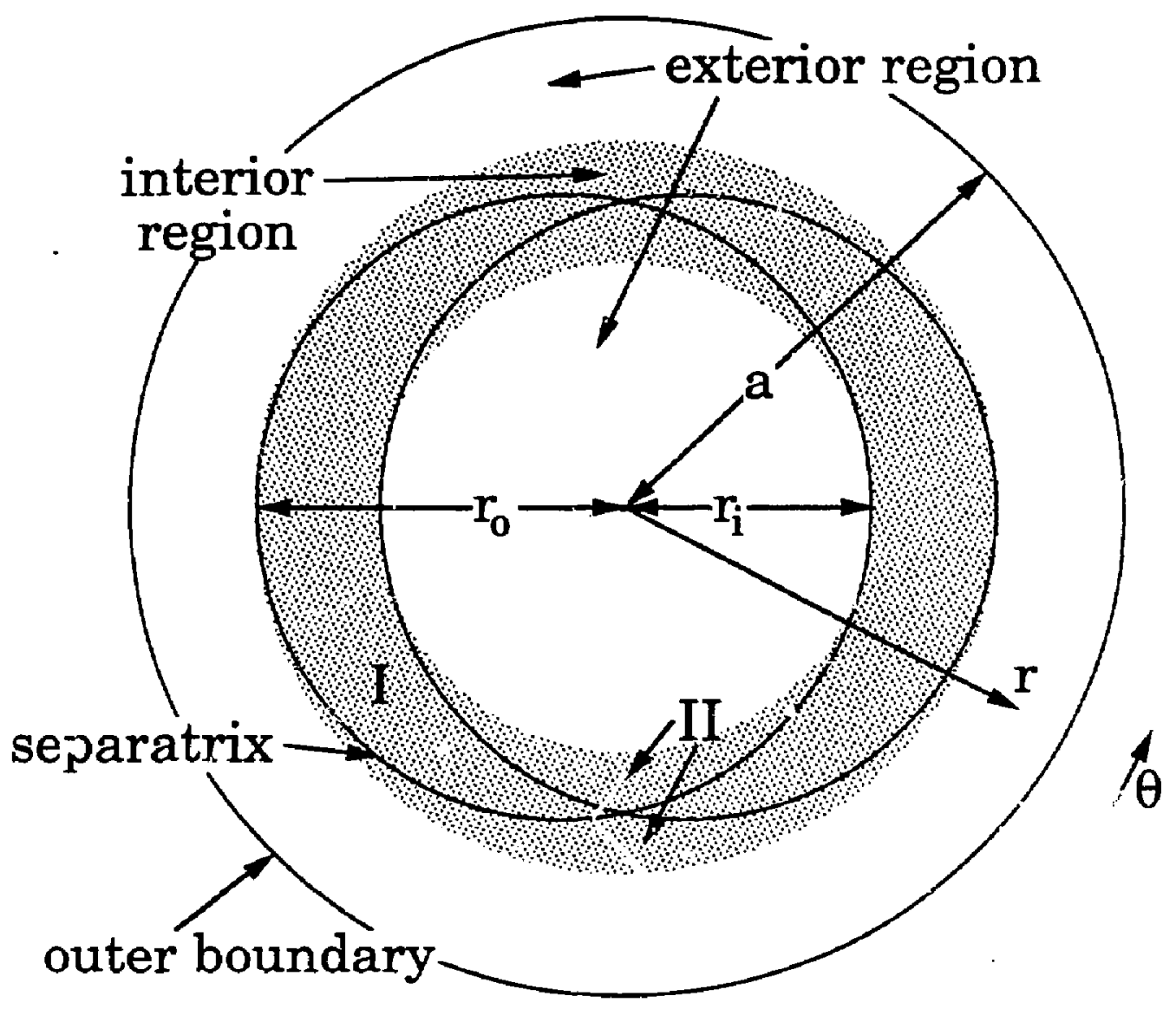

FIG. 1 
$91 \mathrm{~T} 1002$

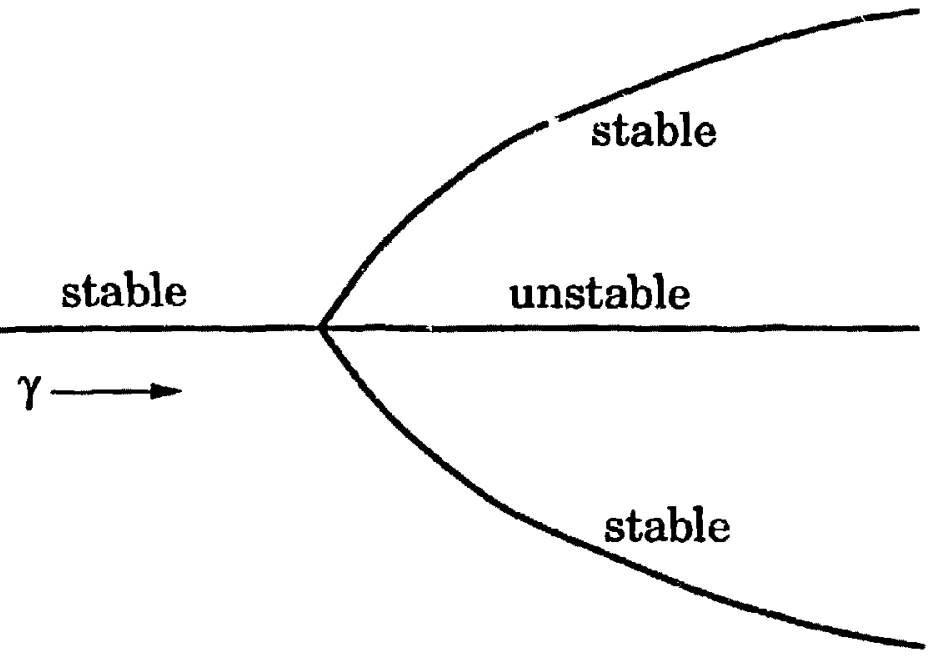

FIs. 2 


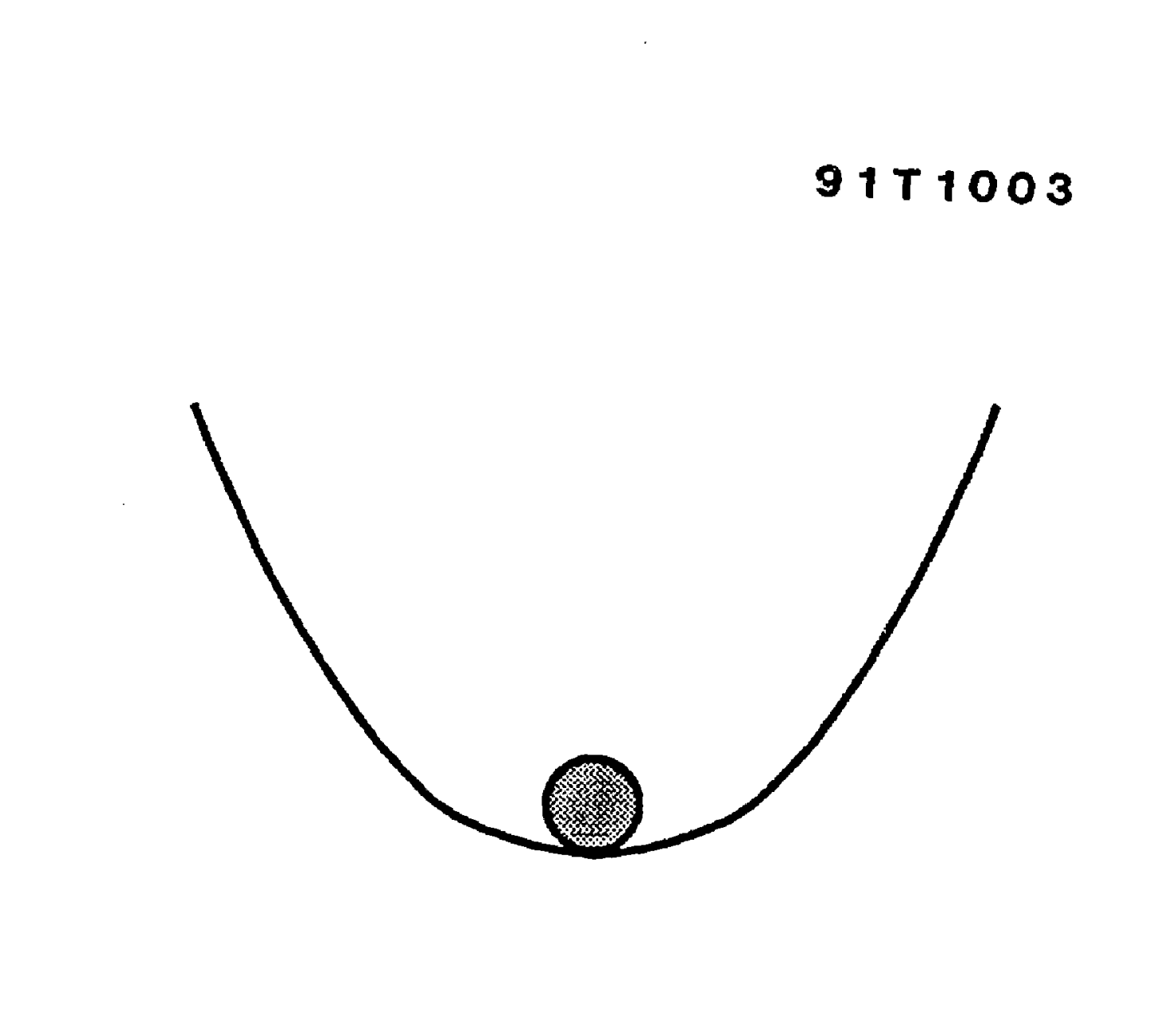


9171004

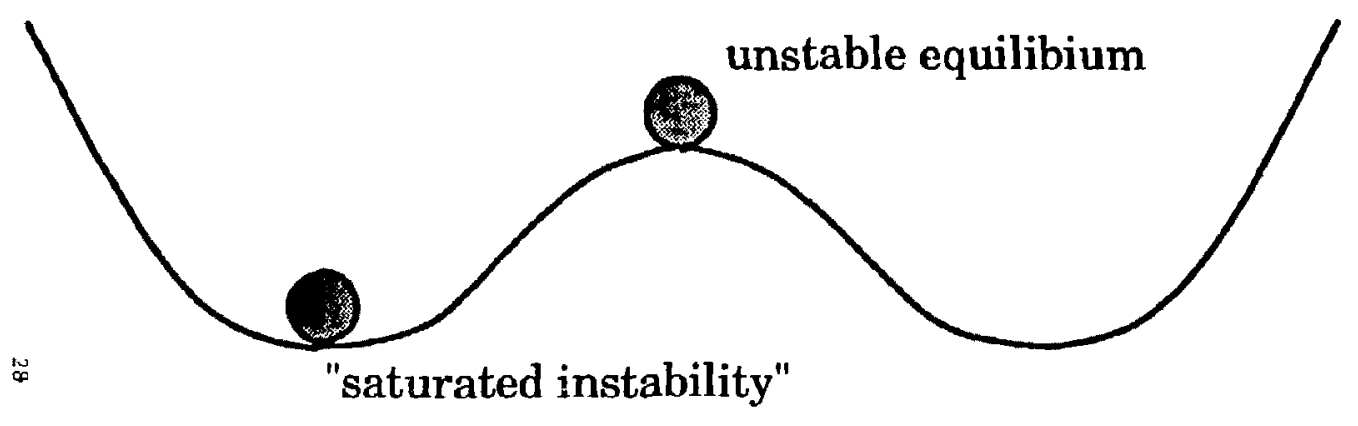

FIG. 4 


\section{$91 \mathrm{~T} 1005$}

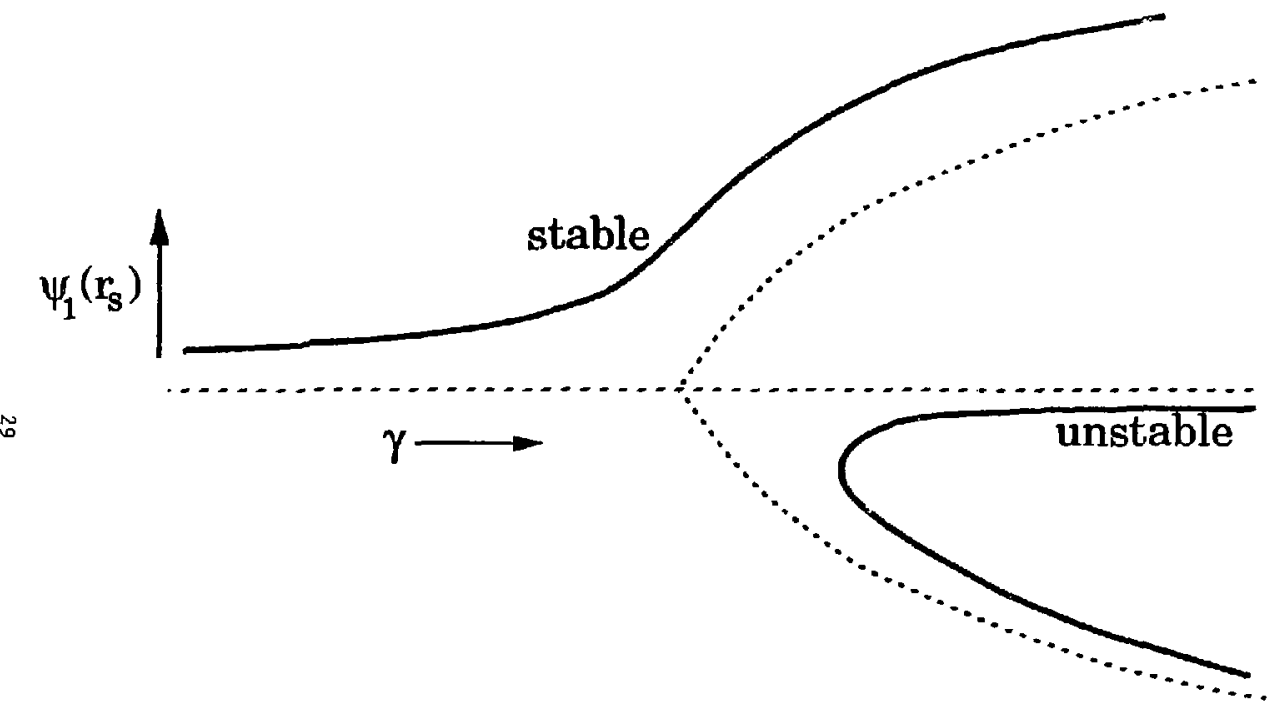

FIs. 5 


$$
d
$$




\section{$91 \mathrm{~T} 1007$}

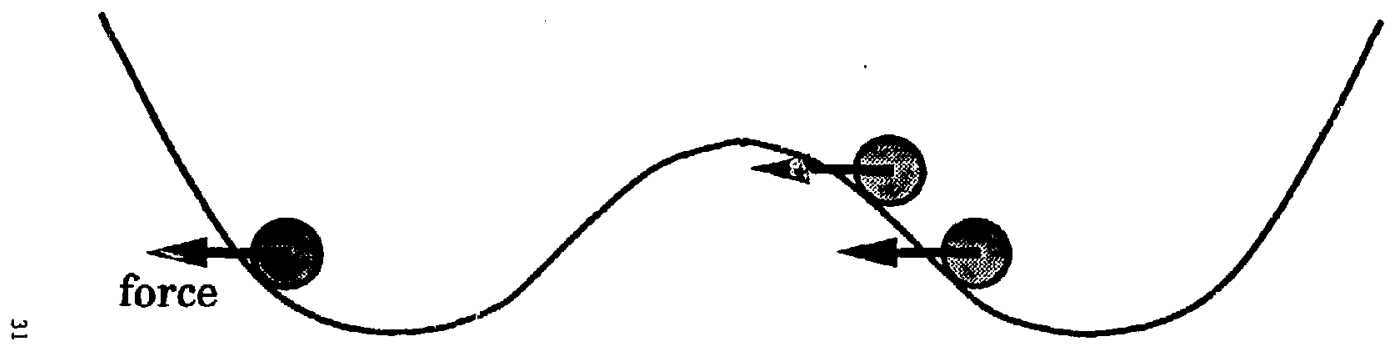

FIG. 7 
$01 T 1008$

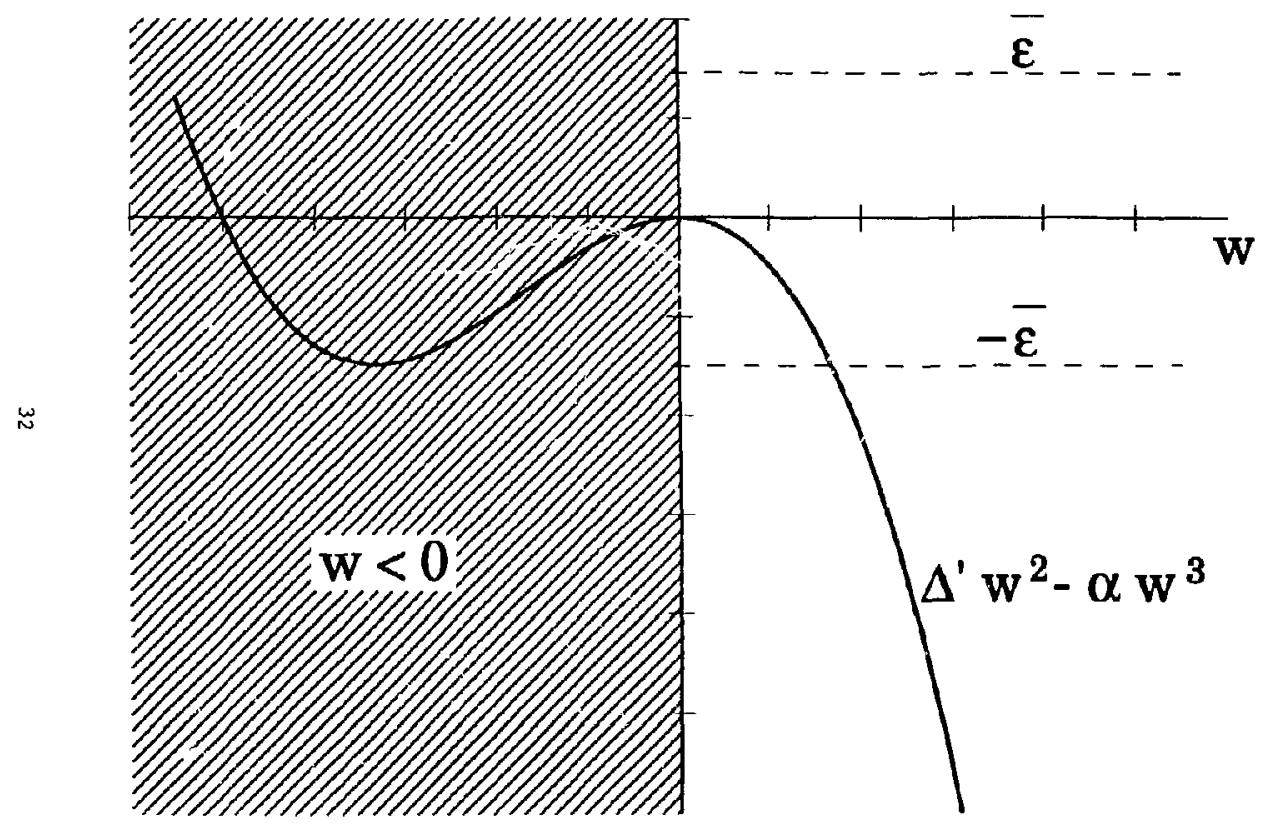

FIG. 8 
$91 T 1009$

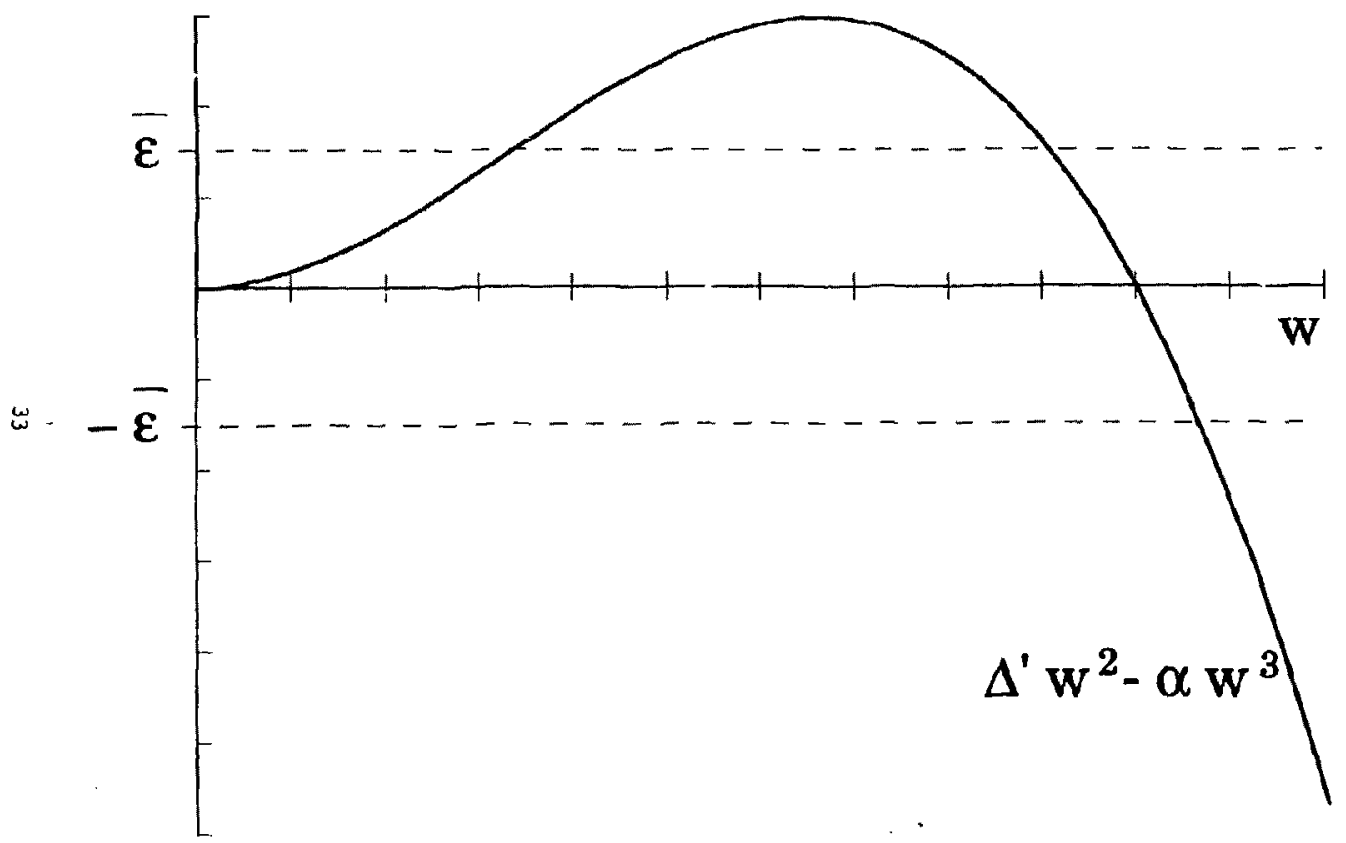

FIT. 9 
$81 T 1010$

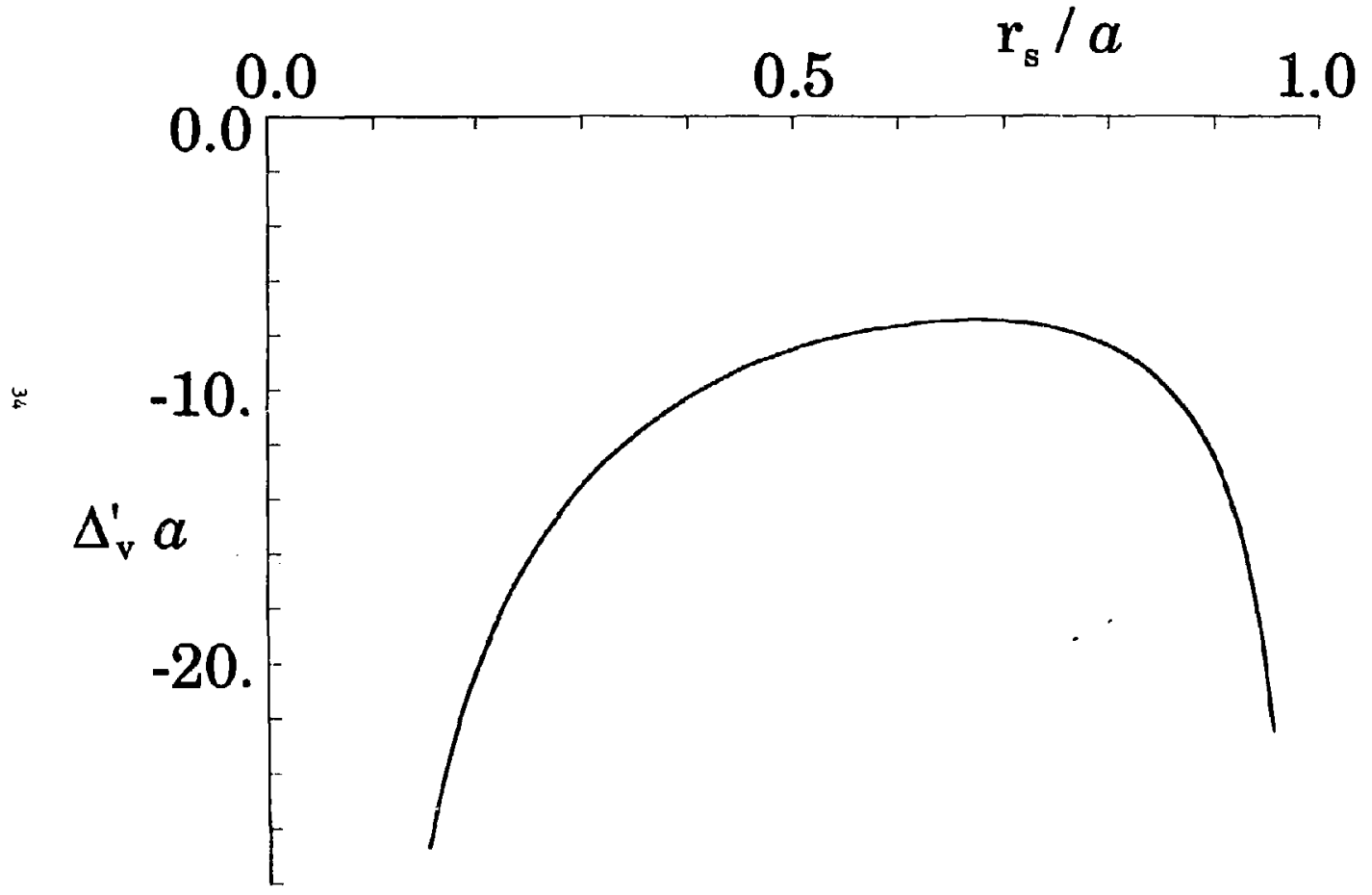

FIG. 10 
91T1011

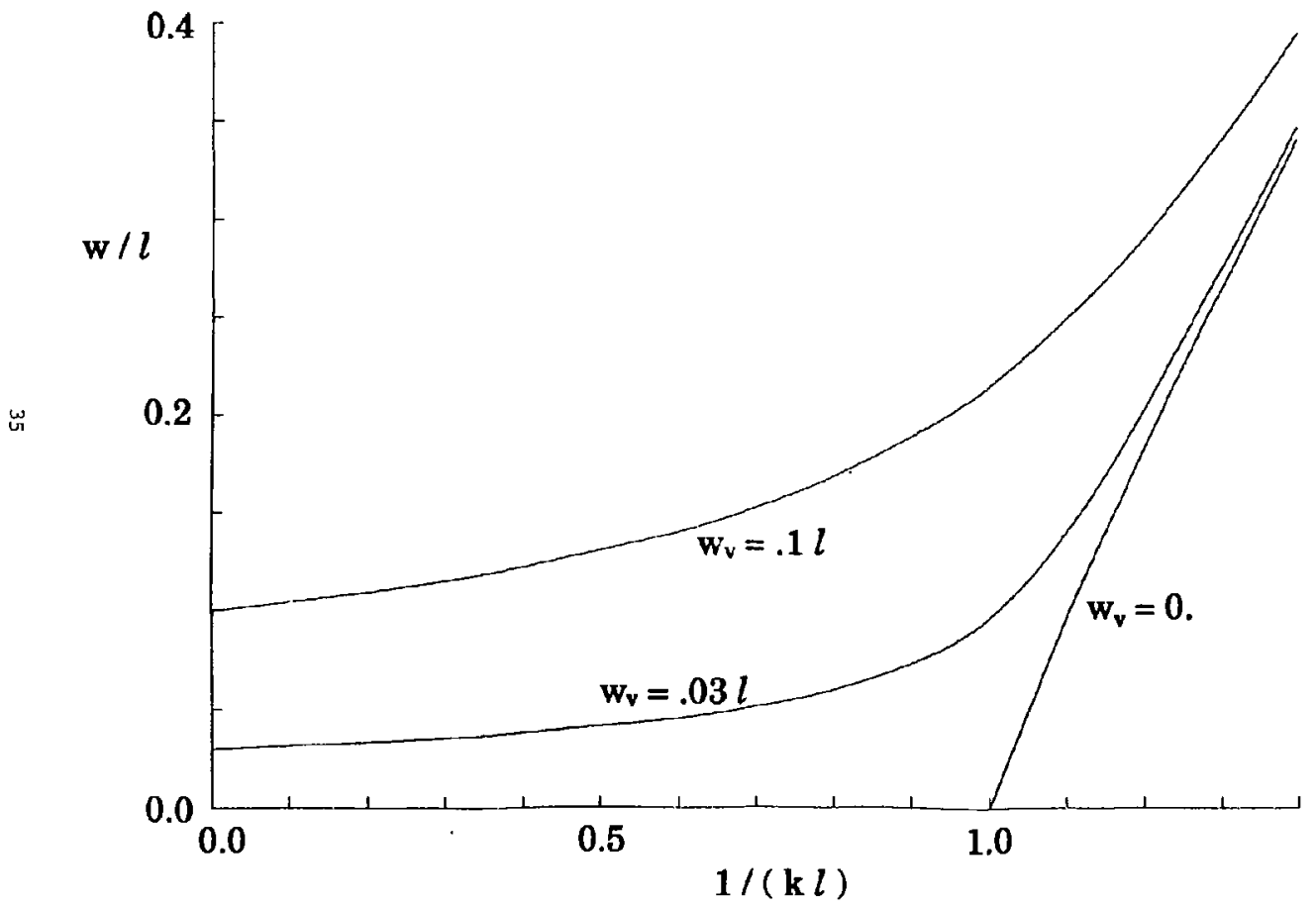

FIC. 11 
a) conventional time-dependent calculation

island
width

profile parameter

b) equilibrium calculation

island
width

profile parameter

FIG. 12 